\title{
A Case of Sjögren's Syndrome with Pleural Effusion: Difficult to Distinguish from Tuberculous Pleurisy Because of a High Adenosine Deaminase Level
}

\author{
Masafumi Shimoda ${ }^{1}$, Yoshiaki Tanaka ${ }^{1}$, Kozo Morimoto' ${ }^{1}$, Kiyomi Shimoda ${ }^{2}$, \\ Tamiko Takemura ${ }^{4}$, Teruaki Oka ${ }^{3}$, Takashi Yoshiyama ${ }^{1}$, Kozo Yoshimori ${ }^{1}$ and Ken Ohta ${ }^{1}$
}

\begin{abstract}
:
An 84-year-old woman visited our hospital for dyspnea due to right pleural effusion with lymphocytic dominance and a high adenosine deaminase (ADA) level that had been noted 1 month earlier. She was suspected of having tuberculosis pleurisy; however, anti-tuberculosis treatment yielded no improvements. She was diagnosed with pleural effusion due to primary Sjögren's syndrome $(\mathrm{SjS})$ based on her dry eyes and mouth, positivity for anti-Sjögren's-syndrome-related antigen A/B, and histopathologic findings of a lip biopsy and thoracoscopic pleural biopsy. Her symptoms improved after starting steroid therapy. Cases of pleural effusion due to $\mathrm{SjS}$ with a high ADA level may be misdiagnosed as tuberculosis pleurisy.
\end{abstract}

Key words: Sjögren's syndrome, pleural effusion, adenosine deaminase, tuberculous pleurisy

(Intern Med Advance Publication)

(DOI: 10.2169/internalmedicine.7818-21)

\section{Background}

Primary Sjögren's syndrome $(\mathrm{SjS})$ is a chronic autoimmune disorder of the exocrine glands with associated lymphocytic infiltration of the affected glands; $\mathrm{SjS}$ is a rare disease, with a prevalence in the general population of approximately $0.5 \%$ (1).

Pleural effusion is very rare in $\mathrm{SjS}$ patients, with an incidence of less than $1 \%$ (2). Some studies on pulmonary manifestations of primary $\mathrm{SjS}$ have not reported pleural effusion (2), and a previous report stated that only 2 of 343 patients with primary $\mathrm{SjS}$ had pleural effusion (3). Generally, pleural effusion in patients with $\mathrm{SjS}$ is exudative with lymphocytic dominance, a high concentration of antiSjögren's syndrome-related antigen A (SS-A)/anti-Sjögren's syndrome-related antigen B (SS-B), low complement levels, and usually low adenosine deaminase (ADA) levels (4); the relationship between $\mathrm{SjS}$ and pleural effusion is uncertain $(2,5)$. The level of ADA in the pleural fluid is gener- ally used as a marker for a diagnosis of tuberculous pleurisy $(6,7)$.

We herein report a rare case of $\mathrm{SjS}$ with high $\mathrm{ADA}$ levels in the pleural effusion that was distinguished from tuberculous pleurisy based on a thoracoscopic pleural biopsy.

\section{Case Presentation}

We encountered an 84-year-old woman who had developed dyspnea 1 month earlier. She had a medical history of bronchial asthma, eosinophilic pneumonia, and diabetes mellitus. She had no smoking history and no relevant allergic history. She visited a local doctor due to dyspnea, and radiography showed the presence of right pleural effusion. Thoracentesis revealed that her pleural effusion was exudative with lymphocytic dominance and high ADA levels of $52 \mathrm{U} /$ L; therefore, she was suspected of having tuberculosis pleurisy and visited our hospital.

Her vital signs were normal, although she had a lowgrade fever of $37.3{ }^{\circ} \mathrm{C}$. A physical examination revealed de-

\footnotetext{
${ }^{1}$ Respiratory Disease Center, Fukujuji Hospital, Japan Anti-tuberculosis Association, Japan, ${ }^{2}$ Department of Thoracic Surgery, Fukujuji Hospital, Japan Anti-tuberculosis Association, Japan, ${ }^{3}$ Department of Pathology, Kanagawa Cardiovascular and Respiratory Center, Japan and ${ }^{4}$ Department of Pathology, Fukujuji Hospital, Japan Anti-tuberculosis Association, Japan

Received: April 23, 2021; Accepted: June 28, 2021; Advance Publication by J-STAGE: August 13, 2021

Correspondence to Dr. Masafumi Shimoda, shimodam@fukujuji.org
} 

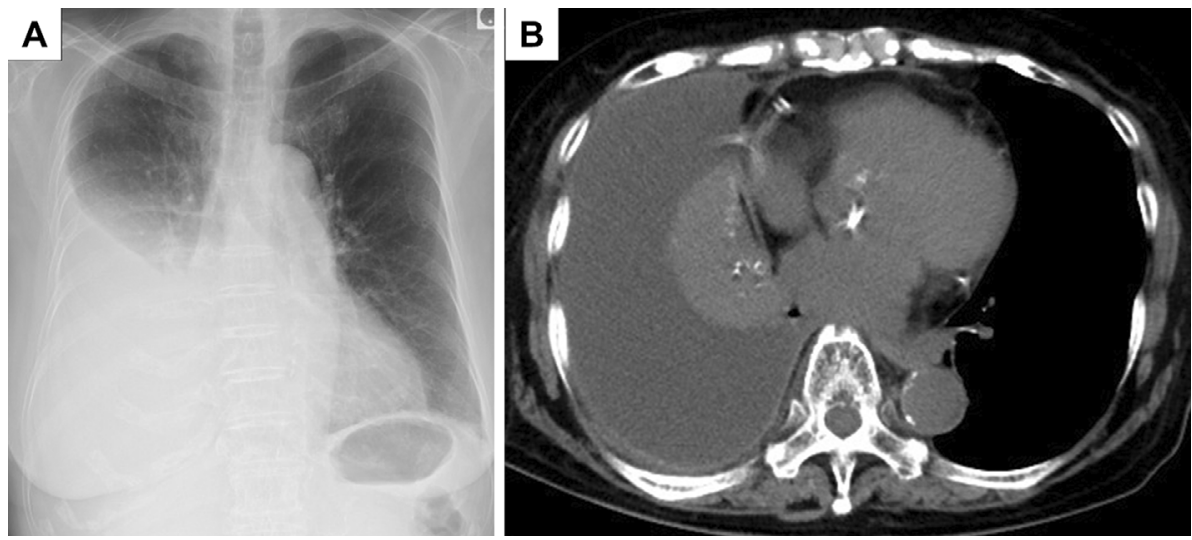

Figure 1. Chest radiography and computed tomography (CT) showed a large amount of right pleural effusion without a lung lesion.
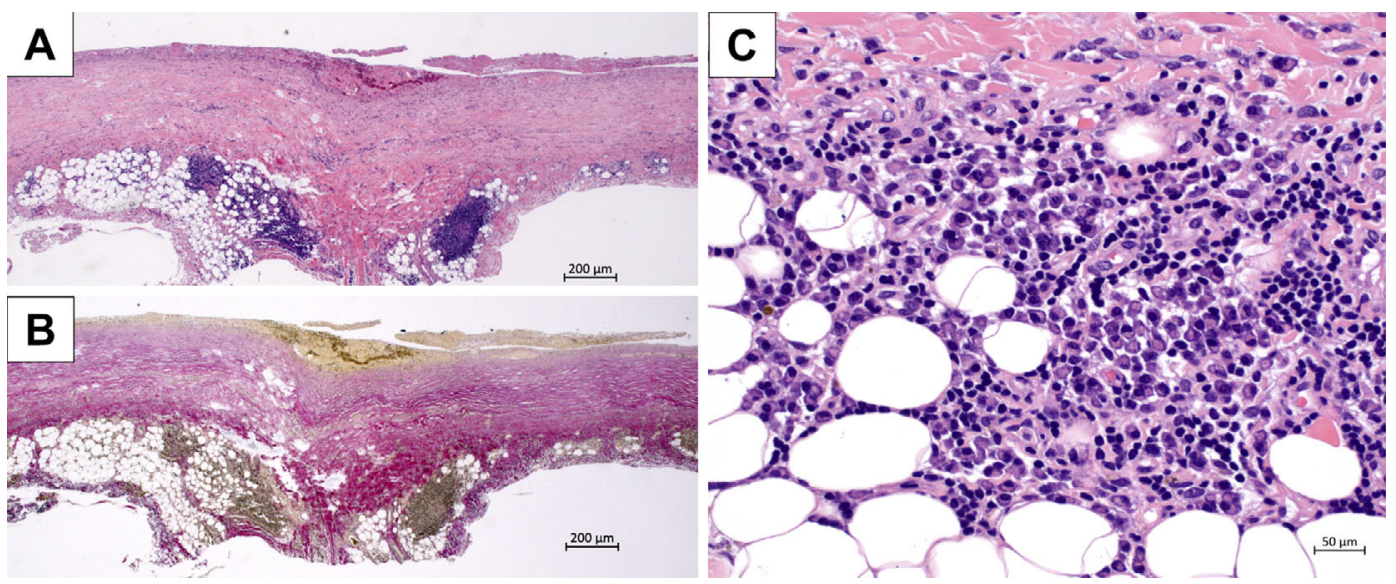

Figure 2. A thoracoscopic pleural biopsy revealed pleural fibrotic thickening with marked infiltration of lymphocytes, without evidence of granuloma formation or malignancy. A: Hematoxylin and Eosin $($ H\&E) staining $\times 50$. B: Elastica van Gieson staining $\times 50$. C: $\mathbf{H} \& E$ staining $\times 200$.

creased breath sounds in the right lower lung, dry eye, and dry mouth. Laboratory findings were as follows: white blood cell count of 4,620 cells $/ \mu \mathrm{L}$, with $81.0 \%$ polymorphic nuclear leukocytes; C-reactive protein level of $3.94 \mathrm{mg} / \mathrm{dL}$; hemoglobin A1c level of 8.4\%; antinuclear antibody (ANA) 1:160 dilution with speckled immunofluorescence pattern; anti-SS-A antibody level $>1,200.0 \mathrm{U} / \mathrm{mL}$; and anti-SS-B antibody level of $563.0 \mathrm{U} / \mathrm{mL}$. Her serum $\mathrm{IgG}$ and $\mathrm{IgG} 4$ levels were $2,651 \mathrm{mg} / \mathrm{dL}$ and $39.4 \mathrm{mg} / \mathrm{dL}$, respectively. Anticyclic citrullinated peptide antibody and an interferon gamma release assay (IGRA) showed negative results.

Chest radiography and computed tomography (CT) showed a large amount of right pleural effusion without a lung lesion (Fig. 1A, B). The pleural effusion obtained by thoracentesis showed lymphocyte dominance (78.0\%), a total protein (TP) level of $5.88 \mathrm{~g} / \mathrm{dL}$, a lactate dehydrogenase (LDH) level of $230 \mathrm{IU} / \mathrm{dL}$, a glucose level of $131 \mathrm{mg} / \mathrm{dL}$, and an ADA level of 45.1 U/L. Pleural fluid bacterial culture, tuberculosis examinations (smear and polymerase chain reaction $[\mathrm{PCR}]$ tests), and cytology were negative.

She was suspected of having tuberculous pleurisy due to her high ADA levels and was started on empiric therapy with antituberculosis medicine (isoniazid, rifampicin, ethambutol, and pyrazinamide). However, her symptoms and pleural effusion did not improve after over two months of treatment. Additional examinations of the pleural effusion showed ANA at a 1:160 dilution with a speckled immunofluorescence pattern, an anti-SS-A antibody level > 1,200.0 U/mL, and an anti-SS-B antibody level of $656.0 \mathrm{U} /$ $\mathrm{mL}$. The pleural fluid $\mathrm{IgG}$ level was $1,965 \mathrm{mg} / \mathrm{dL}$, and the IgG4 level was $21.0 \mathrm{mg} / \mathrm{dL}$. The complement component levels in the pleural effusion (C3 $41 \mathrm{mg} / \mathrm{dL}, \mathrm{C} 45 \mathrm{mg} / \mathrm{dL}$, and total complement $<12.0 \mathrm{U} / \mathrm{mL}$ ) were lower than in the serum (C3 $128 \mathrm{mg} / \mathrm{dL}, \mathrm{C} 416 \mathrm{mg} / \mathrm{dL}$, and total complement $45.9 \mathrm{U} / \mathrm{mL})$.

We performed a thoracoscopic pleural biopsy, which revealed pleural fibrotic thickening with marked infiltration of lymphocytes and no evidence of granuloma formation or malignancy (Fig. 2A-C). Immunohistochemical staining showed few IgG4-positive cells and an IgG4/IgG ratio < $25 \%$. In addition, an ophthalmological examination showed a positive Schirmer test (right eye $0 \mathrm{~mm}$, left eye $0 \mathrm{~mm}$ ), and keratoconjunctivitis sicca was observed. The Gam test showed reduced salivary flow (3.5 mL/10 minutes). A histo- 


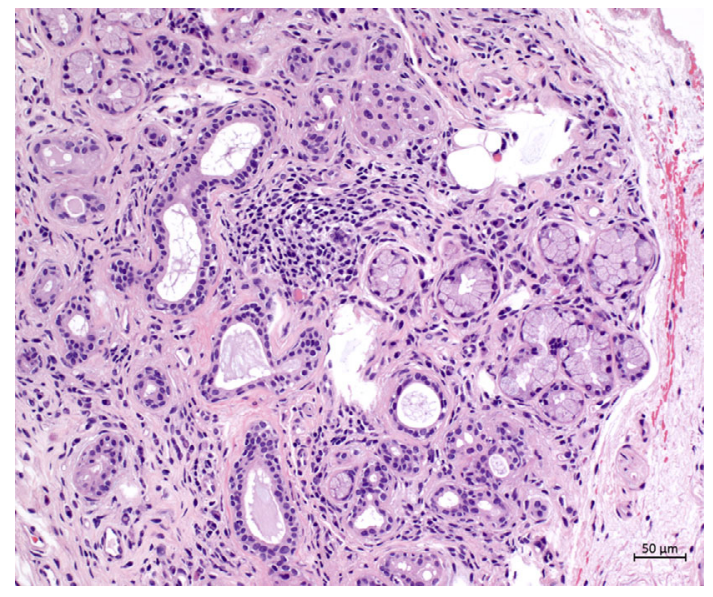

Figure 3. A histopathologic examination of the lip biopsy showed lymphocyte infiltration around the salivary gland ducts.

pathologic examination of the lip biopsy showed lymphocyte infiltration around the salivary gland ducts (Fig. 3). Accordingly, she met the diagnostic criteria for and was diagnosed with pleural effusion due to primary SjS (1).

Her symptoms, laboratory findings and radiographic findings gradually improved after starting treatment with $20 \mathrm{mg}$ of prednisolone. The prednisolone dose was gradually reduced to $9 \mathrm{mg}$ over the course of 6 months, and there has been no evidence of recurrence thus far. We obtained informed consent from the patient for the publication of this report.

\section{Discussion}

We herein report a case of primary $\mathrm{SjS}$ with pleural effusion, characterized by lymphocytic dominance, exudation, and a high concentration of ADA. It was difficult to rule out tuberculous pleurisy; therefore, we performed a thoracoscopic pleural biopsy. The anti-SS-A and anti-SS-B levels in the pleural effusion were elevated, similar to the serum data. Her symptoms, laboratory findings, and radiographic findings improved with steroid therapy.

Generally, SjS patients with pleural effusion show lymphocytic dominance and exudation with normal glucose levels and $\mathrm{pH}$ and low pleural fluid ADA levels (2). ADA is an enzyme produced by the lymphocytes that participates in purine catabolism in the pathway from adenosine to inosine (6). Elevated levels of ADA in the pleural fluid are a useful marker for the diagnosis of tuberculous pleurisy $(6,7)$, and the most widely accepted cut-off value for ADA in the pleural fluid for the diagnosis of tuberculous pleurisy is $40 \mathrm{U} / \mathrm{L}$, with a sensitivity and specificity of $92 \%$ and $90 \%$, respectively (7). Therefore, in our patient, tuberculous pleurisy could not be ruled out initially, and empiric therapy with antituberculosis medicine and a thoracoscopic pleural biopsy were needed.

There have been 12 other case reports of primary $\mathrm{SjS}$ with pleural effusion, which are shown in Table $(5,8-18)$.
Five of the 13 total reports, including our study, described the pleural fluid ADA levels, and 3 patients had high ADA levels of $\geq 40 \mathrm{U} / \mathrm{L}(9,10)$. Furthermore, pleural effusion due to rheumatoid arthritis (RA), systemic lupus erythematosus (SLE), and IgG4-related disease (IgG4-RD) can also show lymphocytic dominance and high concentrations of ADA $(19,20)$; therefore, autoimmune diseases with pleural effusion, such as RA, SLE, SjS, IgG4-RD, are difficult to distinguish from tuberculous pleurisy, even if thoracentesis shows a high ADA level.

A review of the literature revealed the characteristics of $\mathrm{SjS}$ with pleural effusion. Nine patients had high concentrations of anti-SS-A and/or anti-SS-B in their pleural effusion, findings that were similar to the serum data. Many patients were positive for serum ANA, and five were positive for ANA in both the serum and pleural effusion. With regard to the laterality of pleural effusion, 7 patients had bilateral effusion (53.8\%), while 6 had unilateral effusion (46.2\%). A previous review reported that pleural involvement in $\mathrm{SjS}$ was more often bilateral than unilateral (2). A pleural biopsy was reported in five case studies, with nonspecific findings noted. The biopsy was used to rule out tuberculous pleurisy and malignancies. Many patients were treated with steroid therapy, three received pulse corticosteroid therapy, and one received intravenous cyclophosphamide. Conversely, one patient was reported to experience natural remission. Another characteristic of $\mathrm{SjS}$ with pleural effusion is that the levels of complement components, such as $\mathrm{C} 3$ and $\mathrm{C} 4$, and the total complement levels in the pleural effusion are low, although the serum complement component levels are in the normal ranges $(4,9,18)$, similar to the findings in our patient. The presence of anti-SS-A and/or anti-SS-B is thought to lead to pleuritis $(5,12)$; high concentrations of anti-SS-A and/or anti-SS-B in the pleural effusion may therefore be important for the diagnosis of $\mathrm{SjS}$. There are no data on anti-SS-A and/or anti-SS-B in the pleural effusion in patients with other autoimmune diseases.

$\mathrm{SjS}$ with pleural effusion is very rare, and the possibility of secondary SjS should be considered (4). Secondary $\mathrm{SjS}$ is often associated with other autoimmune disorders, such as RA, SLE, and systemic sclerosis (1). In particular, the histological features of pleural disease in patients with SLE are lymphocytic and plasma cell infiltration and pleural fibrosis (2), with reduced levels of complement components (2), as in patients with $\mathrm{SjS}$ with pleural effusion $(9,18)$. Conversely, $\mathrm{SjS}$ patients usually have serum complement component levels within the normal ranges $(5,11-14)$, while SLE patients have reduced levels (2). In RA patients, thoracentesis shows low glucose levels in the pleural effusion (2), which is useful for distinguishing RA from $\mathrm{SjS}$. Our patient was diagnosed with primary $\mathrm{SjS}$ because she had serum complement component levels within the normal ranges and normal glucose levels in the pleural effusion; she did not meet the diagnostic criteria for SLE (21) or RA (22). However, the characteristics of pleural effusion in secondary $\mathrm{SjS}$ have rarely been reported, so whether or not pleural effusion 


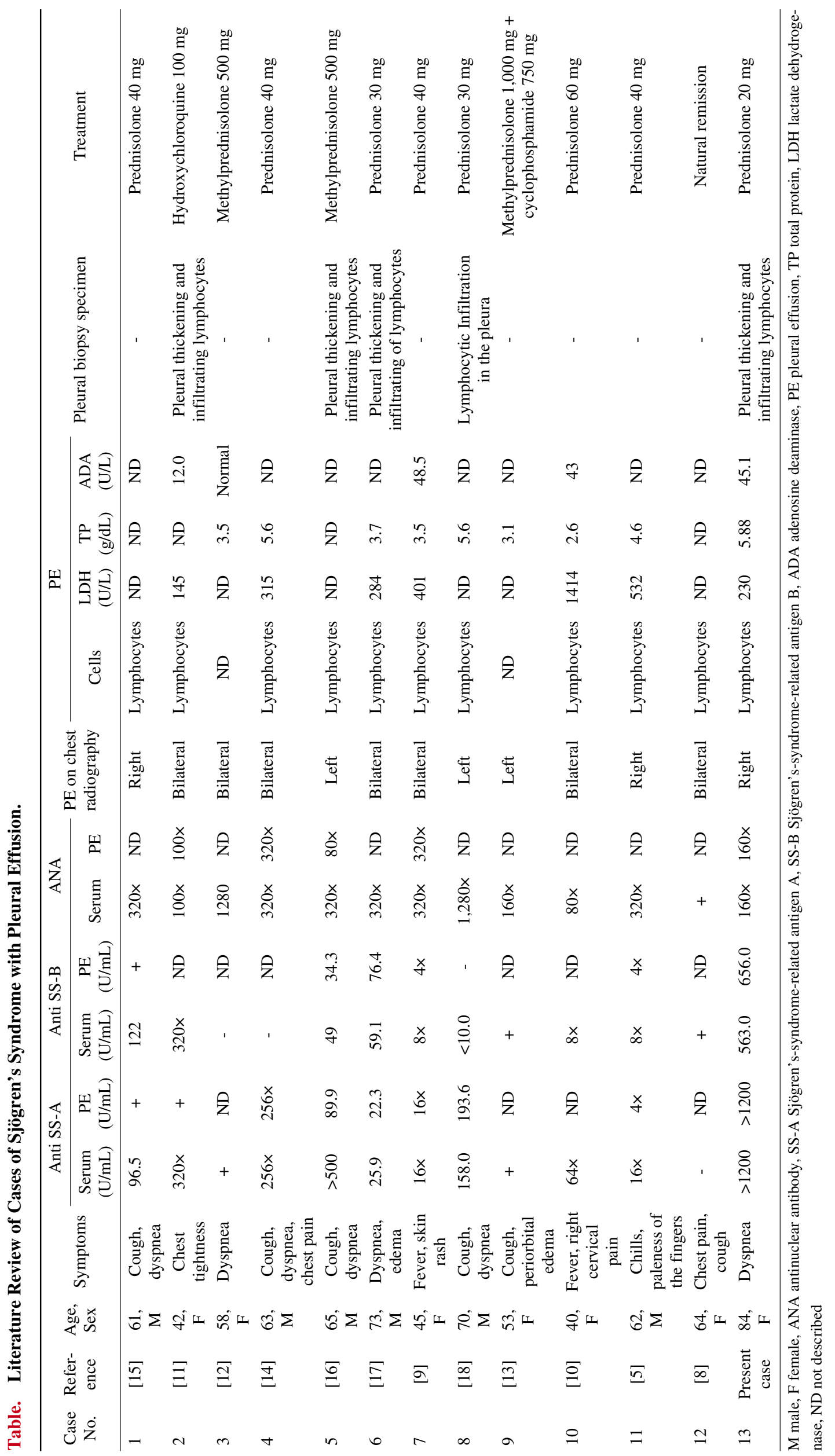


in patients with secondary $\mathrm{SjS}$ is similar to that in patients with SLE or RA is unclear.

\section{Conclusion}

We encountered a rare case of $\mathrm{SjS}$ with pleural effusion, high ADA levels, and positivity for anti-SS-A/anti-SS-B antibodies. It is important to distinguish patients with $\mathrm{SjS}$ with pleural effusion from those with tuberculosis pleurisy.

The authors state that they have no Conflict of Interest (COI).

\section{Acknowledgments}

None

\section{References}

1. Fox RI. Sjogren's syndrome. Lancet 366: 321-331, 2005.

2. Bouros D, Pneumatikos I, Tzouvelekis A. Pleural involvement in systemic autoimmune disorders. Respiration 75: 361-371, 2008.

3. Strimlan CV, Rosenow EC, 3rd, Divertie MB, Harrison EG, Jr. Pulmonary manifestations of Sjogren's syndrome. Chest 70: 354361, 1976.

4. Stojan G, Baer AN, Danoff SK. Pulmonary manifestations of Sjogren's syndrome. Curr Allergy Asthma Rep 13: 354-360, 2013.

5. Ogihara T, Nakatani A, Ito H, et al. Sjogren's syndrome with pleural effusion. Intern Med 34: 811-814, 1995.

6. Aggarwal AN, Agarwal R, Sehgal IS, Dhooria S. Adenosine deaminase for diagnosis of tuberculous pleural effusion: A systematic review and meta-analysis. PLoS One 14: e0213728, 2019.

7. Liang QL, Shi HZ, Wang K, Qin SM, Qin XJ. Diagnostic accuracy of adenosine deaminase in tuberculous pleurisy: a metaanalysis. Respir Med 102: 744-754, 2008.

8. Alvarez-Sala R, Sanchez-Toril F, Garcia-Martinez J, Zaera A, Masa JF. Primary Sjogren syndrome and pleural effusion. Chest 96: 1440-1441, 1989.

9. Tanaka A, Tohda Y, Fukuoka M, Nakajima S. [A case of Sjogren's syndrome with pleural effusion]. Nihon Kokyuki Gakkai Zasshi 38: 628-631, 2000.

10. Kashiwabara K, Kishi K, Narushima K, et al. [Primary Sjogren's syndrome accompanied by pleural effusion]. Nihon Kyobu Shikkan Gakkai Zasshi 33: 1325-1329, 1995.

11. Ma D, Lu H, Qu Y, Wang S, Ying Y, Xiao W. Primary Sjogren's syndrome accompanied by pleural effusion: a case report and literature review. Int J Clin Exp Pathol 8: 15322-15327, 2015.

12. Ohe M, Hashino S, Ohara K. A case of primary Sjögren's syndrome with polyserositis. Eastern Journal of Medicine 19: 54-57, 2014.

13. Suzuki H, Hickling P, Lyons CB. A case of primary Sjogren's syndrome, complicated by cryoglobulinaemic glomerulonephritis, pericardial and pleural effusions. Br J Rheumatol 35: 72-75, 1996.

14. Makimoto G, Asano M, Fujimoto N, et al. Bilateral Pleural Effusions as an Initial Presentation in Primary Sjogren's Syndrome. Case Rep Rheumatol 2012: 640353, 2012.

15. Koga Y, Uchiyama Md K, Tsukamoto Md Ph DN, et al. Primary Sjogren's syndrome with Waldenstrom's macroglobulinemia presenting as unilateral bloody pleural effusion. Respir Investig 55: 283-286, 2017.

16. Teshigawara K, Kakizaki S, Horiya M, et al. Primary Sjogren's syndrome complicated by bilateral pleural effusion. Respirology 13: 155-158, 2008.

17. Horita Y, Miyazaki M, Kadota J, et al. Type II diabetes mellitus and primary Sjogren's syndrome complicated by pleural effusion. Intern Med 39: 979-984, 2000.

18. Kawamata K, Haraoka H, Hirohata S, Hashimoto T, Jenkins RN, Lipsky PE. Pleurisy in primary Sjogren's syndrome: T cell receptor beta-chain variable region gene bias and local autoantibody production in the pleural effusion. Clin Exp Rheumatol 15: 193196, 1997.

19. Shimoda M, Tanaka Y, Morimoto K, et al. IgG4-related pleural effusion with high adenosine deaminase levels: A case report and literature review. Medicine (Baltimore) 100: e25162, 2021.

20. Pettersson T, Klockars M, Weber $T$. Pleural fluid adenosine deaminase in rheumatoid arthritis and systemic lupus erythematosus. Chest 86: 273-274, 1984.

21. Aringer M, Costenbader K, Daikh D, et al. 2019 European League Against Rheumatism/American College of Rheumatology Classification Criteria for Systemic Lupus Erythematosus. Arthritis Rheumatol 71: 1400-1412, 2019.

22. Aletaha D, Neogi T, Silman AJ, et al. 2010 Rheumatoid arthritis classification criteria: an American College of Rheumatology/ European League Against Rheumatism collaborative initiative. Arthritis Rheum 62: 2569-2581, 2010.

The Internal Medicine is an Open Access journal distributed under the Creative Commons Attribution-NonCommercial-NoDerivatives 4.0 International License. To view the details of this license, please visit (https://creativecommons.org/licenses/ by-nc-nd/4.0/).

(C) The Japanese Society of Internal Medicine Intern Med Advance Publication 